\title{
ADVANCEMENT OF NANOTECHNOLOGY IN THE FIELD OF MEDICINE
}

\author{
Devika TK \\ Department of Biotechnology \\ School of Life Science \\ Pondicherry University, Kalapet, Puducherry, India
}

\begin{abstract}
Nanotechnology is the study of extremely small structures of size ranging from 0.1 to $100 \mathrm{~nm}$. It is a new field of technological innovation which finds application in human biology and medicine. Nanomedicine is the key aspect of 21st century. The main objective of nanomedicine is the overall monitoring, regulation and improvement of all human biological systems working at molecular level using nanostructures and engineered instruments to achieve medical advantages. Nanomedicine is being applied in various disciplines such as diagnostics, surgery, ophthalmology, neurology, oncology, drug delivery etc. Numerous nanocarriers have been used for the diagnostics purposes like quantum dots, nanoparticles, dendrimers, carbon nanotube, nanoshells, nanocrystals etc. However, the usage of nanomaterial also encounters various environmental and societal challenges, particularly in regard to toxicity. This review aims to highlight the major contributions of nanotechnology to modern medicine, opportunities, challenges in this field and also on the environmental and societal aspects of their use.
\end{abstract}

Keywords - Nanotechnology, Nanomedicine, contrast agents, drug delivery, Dendrimers, Magnetic Resonance Imaging, Quantum dots, Toxicity

\section{INTRODUCTION}

Advancement in the field of nanotechnology has revolutionized the twentieth century. It has wide applications in the field of medicines and pharmaceuticals. Nanotechnology is the study of extremely small structures (Nanoparticles) of size ranging from 0.1 to $100 \mathrm{~nm}$ (Figure 1). An ideal nanoparticles should have different properties such as magnetism, chemical reactivity, electrical conductance, optical effects and physical strength, from bulk materials as a result of their small size. Nanotechnology involve either top down approach (reducing the size of large structures to smallest structure) or the bottom up approach (involves changing individual atoms and molecules into nanostructures) [1]. Modern nanotechnology is an interdisciplinary science that deals with smallest particles and their special physical, chemical and mechanical properties. Hence nanotechnology is an assembly of physics, biology, chemistry, medicine, electronics and information technology

\author{
Velvili S \\ Department of Biotechnology \\ School of Life Science \\ Pondicherry University, Kalapet, Puducherry, India
}

[3]. Nanotechnology covers numerous disciplines like medicine, engineering, environment, energy, information and communication etc. Nanotechnology when applied to medicine, it is generally termed as "nanomedicine". Different ascepts of nanomedicine includes diagnosis, treatment and assessment. Nanomedicine makes use of diverse nanomaterials such as nanotubes, nanoscalemicrofabricated devices, nanofibers, polymeric nanoconstructs and nanomachines mainly applied to four major medical arenas i.e., theranostics, diagnostics, targeted drug delivery and regenerative medicine. Theranostics is a fusion of words therapy and diagnostics. It is deals with diagnosis and therapy of the disease before it manifests its symptoms in the human body. Diagnostics mainly involves invitro diagnosis (DNA chip, biosensors), in vivo diagnostics and imaging techniques. Targeted drug delivery helps in proper drug delivery and in the treatment of diseases. It helps in efficient targeting and delivery of drugs to specific sites and thereby minimize side effects and dose. Regenerative medicine includes tissue engineering, cell repair etc [7]. Usage of nanomaterial also encounters various environmental and societal challenges, particularly in regard to toxicity [10]. This review aims to highlight the major contributions of nanotechnology to modern medicine, future perspectives of nanomedicine and also on the environmental and societal aspects of their use.

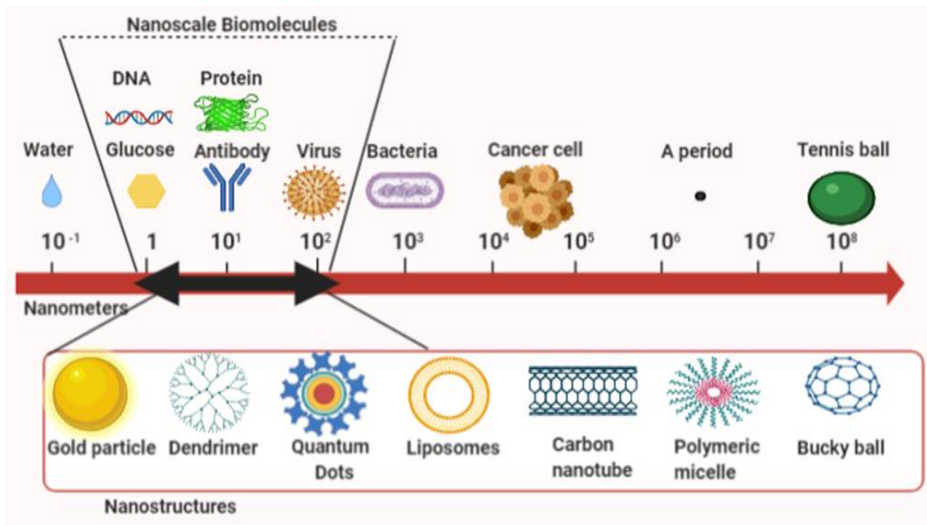

Fig 1: Nanoscale Biomolecules and Nanostructures 


\section{International Journal of Engineering Applied Sciences and Technology, 2020 \\ Vol. 5, Issue 7, ISSN No. 2455-2143, Pages 198-209 \\ Published Online November 2020 in IJEAST (http://www.ijeast.com)}

\section{HISTORY OF NANOTECHNOLOGY}

Nanomedicine is a budding science with lot of tremendous potential [3]. For nanomedicine, nanobiotechnology knowledge which investigates the structure and function of cells, cell communication as well as intracellular and intercellular processes is of prime importance [3]. The major development in the field nanotechnology is mentioned in Table 1.

\begin{tabular}{|l|l|}
\hline Year & \multicolumn{1}{|c|}{ Development in the field of Nanotechnology } \\
\hline 1902 & $\begin{array}{l}\text { Structures smaller than 4 nanometers detected in ruby glasses by } \\
\text { Richard Zsigmondy and Henry Siedentopf }\end{array}$ \\
\hline 1931 & $\begin{array}{l}\text { Transmission electron microscope (TEM) by Max Knoll and Emst } \\
\text { Ruska }\end{array}$ \\
\hline 1936 & $\begin{array}{l}\text { Insight into the atomic range with the field electron microscope by } \\
\text { Erwin Müller }\end{array}$ \\
\hline 1951 & Development of field ion microscope (FIM) \\
\hline 1959 & Concepts of nanotechnological approach by Richard Feynman \\
\hline 1960 & Peter Paul Speiser-first nanoparticles used for targeted drug therapy \\
\hline 1974 & The term nanotechnology -by Taniguchi for the first time. \\
\hline 1981 & $\begin{array}{l}\text { Gerd Binnig and Heinrich Rohrer - scanning tunneling microscope } \\
\text { (STM) }\end{array}$ \\
\hline 1985 & "Bucky Ball" \\
\hline 1986 & First atomic force microscope (AFM) was commissioned \\
\hline 1989 & IBM logo was made \\
\hline 1991 & Carbon Nano tube for the first time by S. Ijima. \\
\hline 1999 & Book by R. Freitas "Nano medicine" was published \\
\hline 2000 & R.A. FreitasJr- "nanodentistry" \\
\hline 2001 & National Nanotechnology Initiative launched. \\
\hline 2003 & Nanotechnology Research and Development Act \\
\hline 2004 & First policy conference on advanced nanotech \\
\hline $2005-$ & 3D Nano systems were prepared \\
\hline 2010 & Era of molecular nano technology started \\
\hline 2011 & Shrinking objects to the nanoscale. \\
\hline 2012 & NNI launched two more Nanotechnology Signature Initiatives (NSIs) \\
\hline 2016 & $\begin{array}{l}\text { Received Nobel Prize in Chemistry ( for design and synthesis of } \\
\text { molecular machines) }\end{array}$ \\
\hline 2017 & Nobel Prize in Physics 2017: Gravitational waves. \\
\hline 2018 & $\begin{array}{l}\text { tic-tac-toe game board made with DNA. } \\
\text { Shrink }\end{array}$ \\
\hline
\end{tabular}

Table 1: Developments in Nanotechnology $[1,2,3,17]$

\section{NANOCARRIERS USED IN NANOMEDICINE}

Numerous nanocarriers have been used for the diagnostics purposes like quantum dots, nanoparticles, dendrimers, carbon nanotube, nanoshells, nanocrystals etc (Figure 2) $[1,7]$.

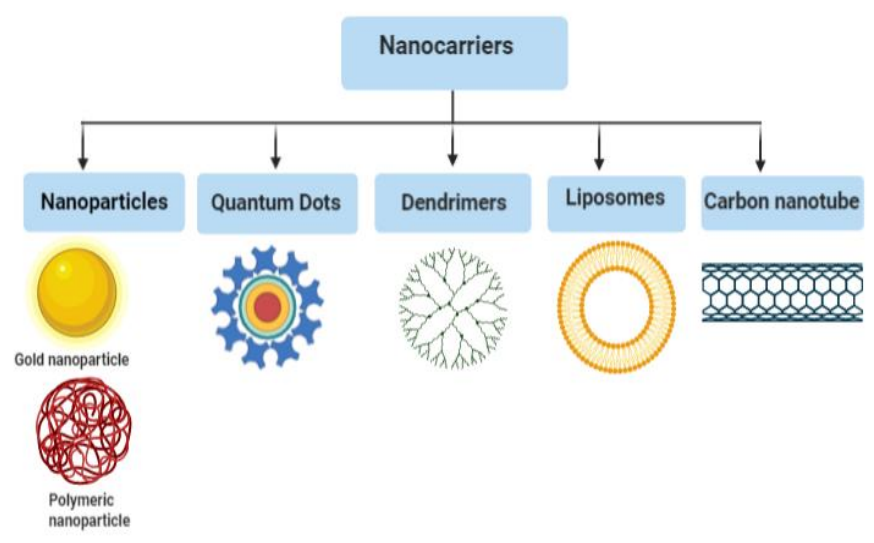

Fig 2: Various nanocarriers used in diagnostics

Nanoparticles (NPs): Nano particle forms the core of nanobiomaterial [15].Nanoparticles are small spheres of size ranging from $10-1000 \mathrm{~nm}$. Nanoparticles are of various types depending upon the nature of material used, for instance, polymeric, gold, superparamagnetic etc. Polymeric nanoparticles are composed of different biodegradable polymers like PLGA, chitosan, sodium alginate etc and are used massively for diagnostic purposes. Modified polymeric nanoparticles are used as contrast agents in Magnetic Resonance Imaging, Computed Tomography etc [7]. Metallic nanoparticles are used in biosensors and in drug delivery, especially in treatment of cancer [1].Superparamagnetic nanoparticles are prepared from magnetic materials like nickel, iron, cobalt etc. that basically comprises of iron oxide core with polymer coating [7]. Metal NPs are made of the metals precursors. Gold nanoparticles (AuNPs) consist of a thin gold shell which surrounds a dielectric core known as nanoshell [7]. Ceramics NPs are inorganic, nonmetallic solids. These nanoparticles have important application in catalysis, photocatalysis, photodegradation of dyes and imaging applications. Semiconductor materials possess properties between metals and nonmetals and therefore they found various applications due to this property [16]. Nanoparticles can be used to increase the bioavailability of various natural compounds [23].

Quantum Dots (QDs): These are 2-10 $\mathrm{nm}$ sized semiconductor nanocrystals. They have quantum confinement properties. QDs emit stable fluorescence from visible to infrared region when it gets excited by light. QDs are composed of positive charged holes and negative charged carriers (electrons). The mechanism of fluorescence is based on the fact that when light of higher energy falls on it, new charged carriers are formed. These newly charged carriers returns to ground state with electrons and holes present in QDs. QDs are mostly composed of two major atoms i.e. cadmium and selenium. It is used in making biosensors [7]. 


\section{International Journal of Engineering Applied Sciences and Technology, 2020 \\ Vol. 5, Issue 7, ISSN No. 2455-2143, Pages 198-209 \\ Published Online November 2020 in IJEAST (http://www.ijeast.com)}

Carbon Nanotubes: These are small macromolecules that have unique importance for biomedical use [1]. It is a hexagonally well ordered nanostructure product which is a carbon allotrope made up of graphite. The length to diameter ratio of carbon nanotubes is more than 106 . The diameter of carbon nanotube is in nanometer range where as its length ranges from nanometers to several millimeters. Nanotubes can be combined with portable Raman probes for theranostic applications [7].

Liposomes: Liposomes are the most extensively explored and developed nanocarriers for novel and targeted drug delivery due to their small size ranging from 50-200 nm. When dry phospholipids are hydrated, closed vesicles are formed. Liposomes are versatile, biocompatible and have good entrapment efficiency. It is mostly used for long circulatory and in passive and active delivery of gene, protein and peptide [1]

Dendrimers: Dendrimers are hyper branched, tree-like structures which contains three different regions: core moiety, branching units and closely packed surface. It has a globular structure and have unique physical properties [1]. Dendrimers have also been used as contrast agents in magnetic resonance imaging (MRI). Dendrimers are not naturally paramagnetic; hence gadolinium or iron oxide must be added to generate MR imaging agents. Dendrimers containing iron oxide nanoparticles are known as magnetodendrimers which are used for labeling and tracking cells [7].

\section{APPLICATIONS OF NANOTECHNOLOGY IN MEDICINE}

The objectives of nanomedicine is to improve human biological systems with the help of nanostructures by monitoring, controlling, constructing, repairing and defensing the system at molecular level. These nanostructures work at cellular level in order to achieve better medical benefits than obtained from usual medical treatment. Various application of nanotechnology in medicine include (Figure 3).

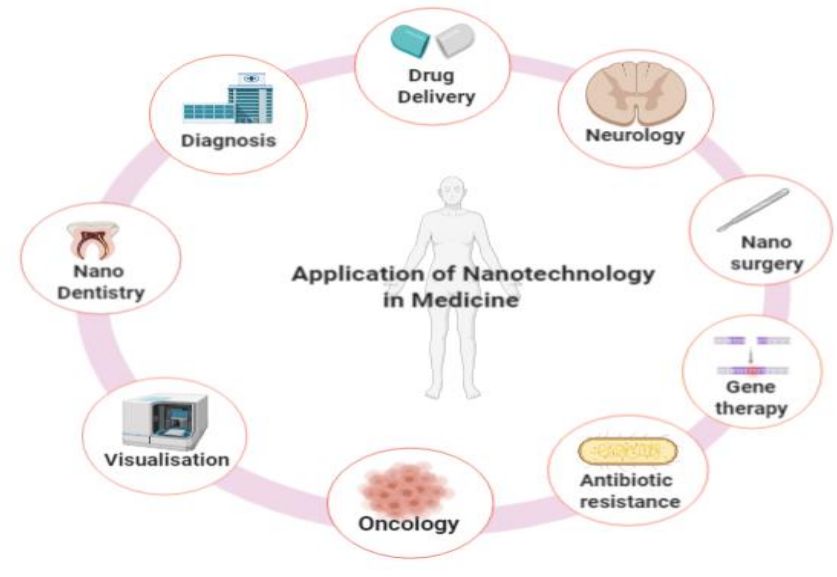

Fig 3: Application of nanotechnology in medicine

\subsection{Nanotechnology in diagnosis}

\subsubsection{In vitro diagnosis}

In vitro diagnostic procedures is a very laborious task as it includes collection of samples (blood, any body fluid or tissues) which are then sent to the laboratory for analysis. This often takes hours, days or even weeks depending on the technique used and it is highly labour intensive. Some of the disadvantages associated with in vitro diagnostic procedures includes sample deterioration, cost, lengthy waiting times (even for urgent cases), inaccurate results for small sample quantities, difficulties in integrating parameters obtained by a wide variety of methods and poor standardisation of sample collection etc [4]. Several single nucleotide polymorphisms (SNPs) are found to exist in the human genome and the corresponding protein variants may differ from each other in a single amino acid and protein also exhibit considerable difference in their functionality. These SNPs are the major cause for various genetic disorders. Invention of DNA chips has made it easier for analysing the DNA for these SNPs. The DNA chip mainly consists of an inert support which carries microarrays of thousands of single stranded DNA molecules with different base sequences. The isolated DNA from a tissue sample has been tagged with a radioactive or any fluorescent material and applied on these DNA chips. It can be identified on the basis of the place on the chip where it binds to the chip DNA. Recently nanotechnology has shown its remarkable role in producing the chips and in increasing their detection sensitivity and reliability. Quantum dots are more frequently used in construction of DNA Chips. DNA present in a sample is identified on the basis of its bonding to DNA molecules of a known composition which are embedded in micrometre-sized polymer spheres containing various mixtures of quantum dots, each of which provides a unique spectral bar code (colour code) [8].

\subsubsection{In Vivo Diagnostics and Imaging}

Nowadays, Molecular imaging has become a basic tool for monitoring diseases. The main drawback of imaging techniques is that it could detect changes in the appearance of tissues only when symptoms are relatively advanced. Later, various contrast agents were introduced to precisely identify and map the locus of a disease [4]. Nanomedicine mainly focus on the use of nanomaterials as contrast agents for anatomical and functional imaging. It enables visualization of structures inside the human body and also helps clinicians to delineate healthy from diseased tissues and to recommend proper treatment for the condition. The most commonly used imaging methods involve Computed Tomography (CT), Magnetic Resonance Imaging (MRI), Positron Emission Tomography (PET) or Single Photon Emission Computed Tomography (SPECT); fluorescence imaging and 


\section{International Journal of Engineering Applied Sciences and Technology, 2020 Vol. 5, Issue 7, ISSN No. 2455-2143, Pages 198-209 \\ Published Online November 2020 in IJEAST (http://www.ijeast.com)}

photoacoustic imaging [5]. Super Paramagnetic Iron Oxide Nanoparticles (SPIONs) were the first nanoparticle constructs to allow molecular imaging used for contrast generation with magnetic resonance imaging (MRI) [11].

Nanoparticles for magnetic resonance imaging: MRI is one of the most powerful tool in molecular imaging. It is a noninvasive technique which provides physiological, anatomical and molecular information within living subjects [5,10]. It has mainly been used for detecting tumours, for imaging the major organs such as the brain and other parts of the central nervous system and for assessing the function of organs such as the heart and kidneys. MRI has high resolution, unlimited penetration depth and excellent soft tissue contrast. An example of conventional contrast agent is paramagnetic complexes (based on $\mathrm{Gd} 3+, \mathrm{Mn} 2+$, or $\mathrm{Fe} 3+$ ) and they are small molecules that leave the vascular system within minutes and are renally cleared. Since the circulation time is short, it is difficult to acquire a high-resolution image of desired sites [5]. Use of suitable contrast agents helps in improving the sensitivity of MRI, which modify the relaxation times of water protons. The paramagnetic gadolinium complexes such as gadolinium diethylenetriamine penta-acetic acid (Gd-DTPA) are the most commonly available MRI contrast agents [12]. Since they have relatively low relaxivity and a high dose of agents is required, they may cause severe side effects such as nephrogenic systemic fibrosis (NSF). Iron oxide-based nanomaterials have been extensively studied as a safe contrast agent to overcome these limitations of gadoliniumbased contrast agents. These iron oxide nanoparticles are mainly cleared by macrophages in the reticuloendothelial system (RES) and slowly degraded in lysosomes. Many iron oxide nanoparticles has been approved by the FDA (US Food and Drug Administration) such as Resovist is used to characterize focal liver lesions in some countries, Ferridex is used as a contrast agent for imaging liver cancer, Ferumoxytol is clinically approved as an MRI contrast agent and for simultaneous treatment of iron deficiency anaemia [12].

Nanoparticles for computed tomography: CT is a noninvasive imaging technique that is commonly used due to its high spatial resolution, low cost, wide availability, rapid image acquisition $[5,12]$. The main imaging principle of CT is based on the X-ray attenuation of human body or contrast agents. Body tissues or contrast agents absorbs or scatters an irradiated X-ray beam and the reduced X-ray intensity is measured by a detector at different angles. Reconstruction of $\mathrm{X}$-ray intensity profiles helps in obtaining 3D whole-body images with very high spatial resolution. In order to acquire high-quality CT images of a region of interest, contrast agents are used. Barium sulfate suspensions and iodinated compounds are the commonly used CT contrast agents. Since these contrast agents has a lot of limitations, nano-sized iodinated contrast agents has been introduced. Nano-sized contrast agents can evade renal filtration so they exhibit a long circulation time which is very important for molecular imaging. Gold nanomaterials can be used as CT contrast agents but there usage is limited as they are too expensive. Tantalum is also used as CT contrast agents owing to its high biocompatibility and radiopacity [12].

Nanoparticles for optical imaging: In Optical imaging techniques, fluorescent colouring agents are taken orally or injected and they accumulate in the tumour. The tumour cells then emit fluorescence when irradiated with laser light. This technique can only be used for imaging tumours that are on or just below the skin or in tissue because the laser light cannot penetrate deep into the body. Intensive research is being carried out into new optical methods based on the use of nanoparticles. Quantum dots are being commonly used as they exhibit excellent optical characteristics including high quantum yield, absorption coefficient and photostability, enabling long-term fluorescence imaging without photobleaching [12]. They are nanoparticles composed of inorganic semiconductor molecules and they emit strong fluorescent light under ultraviolet (UV) illumination. The wavelength (color) of the fluorescent light emitted depends sensitively on particle size [10]. These nanocrystals have the advantage over colouring agents that they fade less quickly over time and do not react with cell components. Moreover, quantum dots of different colours can be made to fluoresce with laser light of the same wavelength, which makes multiplex applications possible. The quantum dots are provided with a layer of lipids or polymers, to prevent heavy metals from being released [8].

\subsection{Nanomedicine and drug delivery}

Dating back to ancient history, it is clear that humans have been using plant based medicinal products for the treatment of various diseases since the past. Natural products have several advantages such as vast chemical diversity, chemical and biological properties with remarkable specificity, low toxicity and side effects. Hence they pave way for the discovery of novel drugs. Computational studies help in developing next-generation drugs which includes targetbased drug discovery and drug delivery [13]. Many potential drug molecules in theory cannot be successfully used as drugs practically. The problem associated with the practical use of those large sized materials in drug delivery includes the following:

(1) Poor solubility: The substance is hardly soluble in water

(2) Poor bioavailability: Before the substance reaches their target site, they are broken down in the body. This breakdown of the substance reduces their bioavailability 


\section{International Journal of Engineering Applied Sciences and Technology, 2020 Vol. 5, Issue 7, ISSN No. 2455-2143, Pages 198-209 \\ Published Online November 2020 in IJEAST (http://www.ijeast.com)}

(3) Poor absorption: Substances find it to difficult to cross certain biological barriers such as cell membranes, blood-brain barrier, placenta.

(4) Non-specific delivery: Due to lack of specificity to the particular target, the substance gets distributed to different kinds of tissues and organs.

These problems can be overruled with the help of nanotechnology which plays a major and successful role in the formulation of drugs and also in their efficient delivery $[8,14]$.

Nanotechnology is a rapidly emerging field of science that has gained interest in recent years to overcome the problems associated with biological and physical sciences, particularly in nanomedicine and nano based drug delivery systems that employs nanoscale range materials [13,14,17]. Nanomedicine and nano delivery systems are relatively new but rapidly developing due to their tremendous potential in molecular diagnostics and drug delivery systems by serving as a means of diagnostic tools or by helping in the controlled delivery of therapeutic agents to specific targeted site, respectively [14]. Nanomedicine has enabled site specific and target oriented delivery of medicines. It has paved for the treatment of several chronic disease, reduces drug consumption and treatment expenses $[1,17]$. Hence in recent times, nanomedicines have become well appreciated [13,14]. Recently for the treatment of various diseases, nanomedicines are being used as biological agents, immunotherapeutic agents, chemotherapeutic agents etc. Nanostructures act as delivery agents by encapsulating drugs or owing to their surface properties therapeutic drugs can be attached to their surface. For instance, for various biomolecule detection procedural assays, gold nanoparticles are used as biomarkers and tumor labels $[13,14]$. Using nanomaterials several anticancer drugs such as paclitaxel, doxorubicin, dexamethasone and 5-fluorouracil have been successfully formulated [13]. For in vitro RNAi delivery, nanoparticles such as Quantom dots, chitosan, Polylactic/glycolic acid (PLGA) and PLGAbased nanoparticles have also been used [13].

Nanomedicine is an emerging field which implements the use of knowledge and techniques of nanotechnology in medical biology, disease prevention and treatment. It utilizes materials of nanoscale range including nanorobots, nanosensors for diagnosis, microfluidics, drug delivery, microarray tests and also actuate materials in live cells. Nanoparticles have wide range of applications, drug delivery is one of the most advanced application of it.

Being nanosized, these structures can be easily taken up by tissues, as a result ensures site specific and target oriented delivery of medicine $[13,14]$. When compared to larger molecules ranging from 1 to $10 \mu \mathrm{m}$, these nanoscale molecules can move freely in the body and their uptake is also much higher. They stay in the circulatory system for longer period of time, as a result help in controlled released of amalgamated drug and [14]. This helps them to directly interact with the diseased cells and treat them with improved efficiency and also helps in reducing the adverse effects [14]. In addition, nanostructures prevent drugs from being tarnished in the gastrointestinal region and also help target specific delivery of sparingly water-soluble drugs. Nanodrugs are taken up by absorptive endocytosis mechanism and hence they show higher oral bioavailability. Nanoparticles are helpful in acquiring information in various novel assays employed to treat and diagnose diseases at all stages of clinical practices.

In recent times, usage of bioactive natural compounds in nanoscience is growing rapidly. Natural compounds can cure various diseases by their ability to act as antimicrobilal agents (Cinnamaldehyde, curcumin) and by inducing tumorsuppressing autophagy (curcumin, caffeine). Besides their characteristic ability, their properties such as bioavailability, targeting and controlled release can be enriched using nanoparticles. For example, thymoquinone obtained from Nigella sativa, when encapsulated in lipid carrier showed six fold increase in bioavailability and increased pharmacokinetic characteristics. Thus it confirms that nanoparticles help in better therapeutic effects of the natural compounds [14].

\subsubsection{Drug designing, drug delivery process and mechanism}

The progression of nanomedicine aims to increase the drug specificity and diagnostic accuracy. This is favoured by the advancement of drug discovery and delivery systems with the help of computational tools and experimental procedures.

While designing target-specific drug delivery systems, drugs with poor solubility and less absorption ability are tagged with nanoparticles (Figure 3) [1,14]. Nanoparticles used in drug delivery systems are usually made up of different biodegradable materials which includes natural or synthetic polymers, lipids, or metals [13]. Besides this, efficacy of drug delivery vehicles depend on biophysical/chemical characteristics such as size, shape etc. An efficient delivery vehicle made up polymeric nanomaterials should range from 10 to $1000 \mathrm{~nm}$ in diameter. Hence compared to larger micromolecules, nanoparticles can be easily taken up by cells due to their smaller size, as a result they can efficiently used in drug delivery systems. Polymeric nanoparticles fall into catergories: Nanopsheres and Nanocapsules. Synthetic polymers (polyvinyl alcohol, poly-l-lactic acid, polyethylene glycol, and poly (lactic-co-glycolic acid) and natural polymers (alginate and chitosan) are extensively used in nanofabrication of nanoparticles due to their high biocompatibility and biodegradability. Polymeric nanoparticles, compact lipid nanostructures and phospholipids (liposomes and micelles) are the most commonly and efficiently used in targeted drug delivery [14]. 


\section{International Journal of Engineering Applied Sciences and Technology, 2020 \\ Vol. 5, Issue 7, ISSN No. 2455-2143, Pages 198-209 \\ Published Online November 2020 in IJEAST (http://www.ijeast.com)}

Based on the physicochemical properties of drugs, appropriate nanoparticles are selected [14]. For therapeutic applications, various nanosystems with different compositions and biological properties are under investigation [13]. For drug delivery purpose, drugs can either be attached to the surface of the nanoparticle or they can be integrated in the matrix of the particle. For efficient drug delivery, the fate of drug which enters the biological environment should be controlled by a drug targeting system [13]. By understanding the interactions of nanomaterials with target cell population through target cell surface receptors, biological environment, drug administration, site of action and mechanism of drug, molecular mechanism behind the pathobiology of the disease under consideration, it is possible to design an effective drug delivery system with the help of nanomaterials. In addition to this it is also important to know the barriers associated with reduced drug efficacy which is mainly attributed to instability of drug inside the cell, multiple targeting or chemical properties of delivering molecules leading to unavailability of drug, changes in genetic makeup of cell-surface receptors or signalling pathways with the progression of disease, over-expression of efflux pumps, drug degradation [13].

The drawback associated with nanomedicine is toxicity of nanoparticles. Hence to overcome the toxicity issues, nanoparticles are used in combination with natural products. Hence nanoparticles synthesized using green chemistry route can minimize side effects of medication. Natural products help in overcoming the challenges associated with drug designing and they also instigate drug discovery with the required physicochemical properties. Bioactivity of the nanomaterials can be increased by adjusting size, shape, hydrophobicity and surface changes of nanostructures [14].

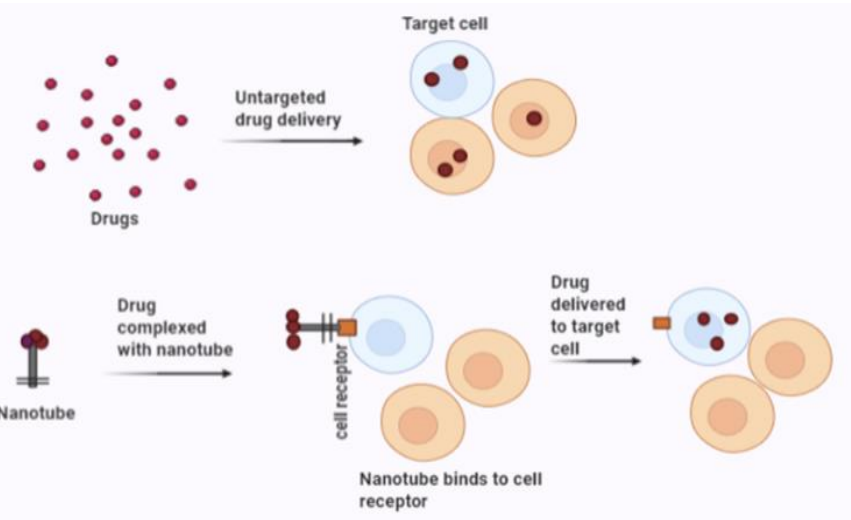

Fig 3: Drug delivery Mechanism (Untargeted and targeted delivery) [14]

\subsubsection{Proteins and peptide delivery}

Biopharmaceuticals include macromolecules such as proteins and peptides, which are used for the treatment of wide range of diseases. This is because under in vivo condition, they have multiple biological actions. For the targeted and controlled delivery of biopharmaceuticals, nanomaterials such as nanoparticles and dendrimers are being used [1].

\subsubsection{Nanoparticles used in drug delivery system}

\section{$\underline{\text { Biopolymeric Nanoparticles }}$}

Biopolymeric nanoparticles are designed using synthetic biodegradable polymers such as polyalkylcyanoacrylate, poly (lactic-co-glycolic acid) and polyanhydride. There are numerous biopolymeric materials with therapeutic potential and hence they are used in drug delivery systems. Despite their therapeutic potential, the stumbling block associated with this is the bionanoparticle-based administration of therapeutic hydrophilic molecules such as peptides, proteins and nucleic acids. This is because the polymers used are hydrophobic where as therapeutic molecules are hydrophilic. Hence it is difficult protect the drugs from enzymatic degradation as it is difficult to encapsulate the drug efficiently with these biopolymers [22].

\section{Protein Nanoparticles}

Two naturally occurring proteins namely albumin and gelatin were first used for designing nanoparticles. These protein based colloid systems had promising potential because they are less immunogenic, no- toxic, biodegradable, highly stable during storage and in vivo condition, can be easily prepared, monitored and scaled up. They also provide opportunities for surface modification and thereby covalently attach to drugs after modification [22].

Albumin: Albumin is a protein found in the blood plasma which has various functions such as transportation, metabolism and distribution of ligands, anti-oxidant. Owing to its function, it is widely used in drug therapy since the past. For instance, literature has marked the use of modified serum albumin as selective agent for the detection of tumor, for the elimination of toxic compounds. Nanomedicine also has made use of the potential of both human serum albumin (HSA) and bovine serum albumin (BSA) as nanoparticle drug targeting carriers, therapeutic enhancers and also as modified vehicles for drug delivery [22].

Collagen: Collagen accounts for nearly $20-30 \%$ of total body protein in the mammalian system. Collagen has magnificent biocompatibility, biodegradation and availability, therefore it is a useful biomaterial. To improve its physicochemical and biological properties, it can be modified easily which paves way for designing various nanoparticles. Collagen based nanoparticles are readily uptaken by the reticuloendothelial system, as result this increases the uptake of drug molecules by the cells [22].

Gelatin: Partial hydrolysis of collagen results in the formation of natural water soluble macromolecule called as 


\section{International Journal of Engineering Applied Sciences and Technology, 2020 \\ Vol. 5, Issue 7, ISSN No. 2455-2143, Pages 198-209 \\ Published Online November 2020 in IJEAST (http://www.ijeast.com)}

gelatin. Gelatin is of two types namely: Type-A gelatin and Type-B gelatin. Gelatin has numerous advantages such as biocompatibility, biodegradation, non-toxic, non-irritablility, low immunogenicity and antigenicity. Because of the qualities which is essential for carrier molecule, it is used in the synthesis of nanoparticles and used in drug delivery for past three decades [22].

Silk proteins: Silk is a natural protein fibres which is made up of two proteins namely fibroin (structural protein) and sericin (gum-like sticky protein). Silk based nanoparticles are widely used in various biomedical applications such as drug or gene delivery, enzyme immobilization, in vivo imaging, etc [22].

Keratin: It is a structural protein rich in cysteine (disulfide bonds), which provides mechanical strength. The keratin nanosuspension coatings are used as alternatives instead of collagen or fibronectin. If explored further, this can also be used in tissue engineering [22].

\section{Polysaccharide nanoparticles}

Nanoparticles obtained from naturally occurring polysaccharides such as chitin, chitosan, hyaluronic acid are used for the delivery of peptides, proteins and nucleic acids. These polysaccharide derived bionanoparticles have helped to improve the biocompatibility of materials toxic to cell with the help of novel immobilization approaches [22].

Alginate: Alginate is a linear, water soluble polysaccharide obtained from brown seaweed. It is made up of two types of uronic acids, $\alpha$-L-guluronic acid and $\beta$-D-mannuronic acid. It has the following properties such as biocompatibility, nonimmunogenic and mucoadhesive in nature. Poly-L-lysine (PLL), a cationic natural polymer is used in combination with alginate to prepare bionanoparticles. But PLL is toxic and immunogenic when injected. Hence another polysaccharide, chitosan is used as an alternative cationic polymer [22].

Chitosan: It is the second abundant naturally occurring polysaccharide made up of $\beta$-(1-4)-linked D-glucosamine and N-acetyl-D-glucosamine. Shells of crabs, shrimps and krill contain chitin, which upon deacetylation yield chitosan. Because of its biocompatibility and biodegradability, it is often used for the microparticles and nanoparticles. Nanoparticles are produced either by spontaneous formation of complexes between chitosan and polyions including DNA or by dispersing chitosan in water-in-oil emulsion which leads to gelation [22].

Other than the above mentioned sources of natural polymers, different other sources such as higher plants, animals, microorganisms and algae are used in nanomedicine (Figure 4).

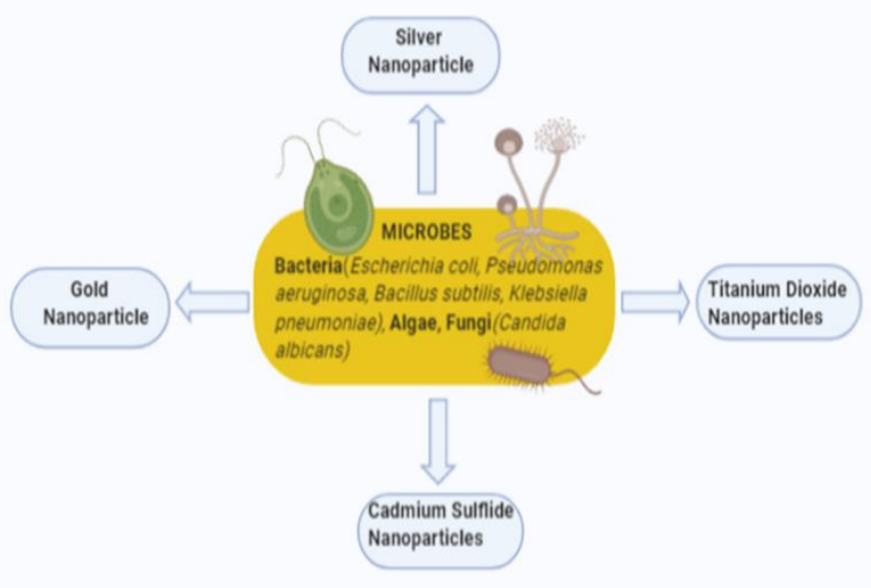

Fig 4: Various nanoparticles synthesised from Microorganisms

\section{Natural product based nanotechnology}

Plants provide ample of natural compounds of therapeutic value which helps in the the discovery of new and highly effective drugs since long time. In today's scenario, number of marketed synthetic molecules are going down and therefore this has lead to more research works on natural product based active compounds. Some of the natural compounds obtained from plants with medicinal value (Figure 5) are as follows: alkaloids, flavonoids, tannins, terpenes, saponins, steroids, phenolic compounds and so on. Besides their medicinal value, these compounds have certain disadvantages which includes high systemic clearance which entails repeated or high doses, low absorption capacity, as a result reduced bioavailability and efficacy. But with the help of nanotechnology, it is possible to solve these problems. Nanotechnology has lead to formulation of various approaches as follows, nanopharmaceutics (treatment with drug), nanodiagnostics (imaging and diagnosis), nanobiomaterial (medical implants) and also combination of diagnosis and treatment known as nanotheranostics[14].

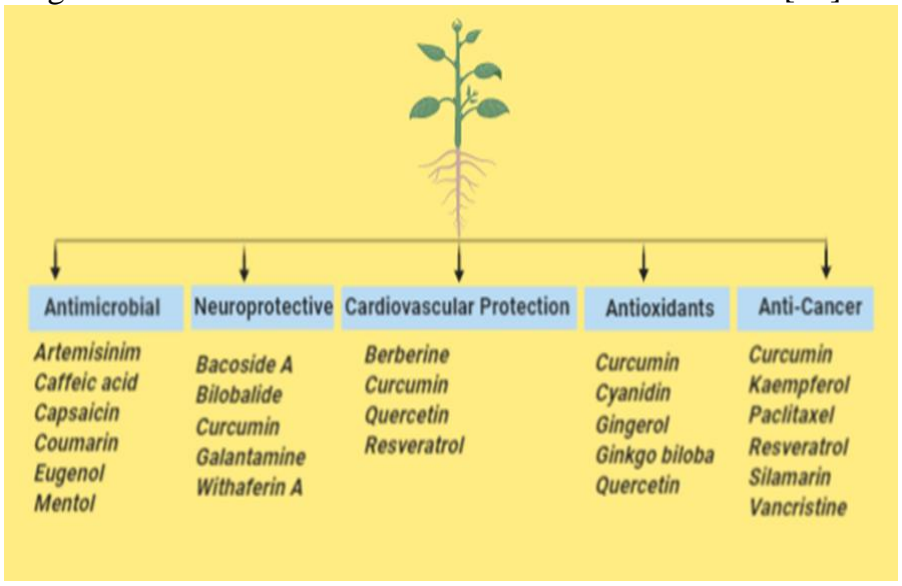




\section{International Journal of Engineering Applied Sciences and Technology, 2020 Vol. 5, Issue 7, ISSN No. 2455-2143, Pages 198-209 \\ Published Online November 2020 in IJEAST (http://www.ijeast.com)}

Fig 5: Natural compounds extracted from higher plants used in nanomedicine [14]

Nanoparticles can be synthesized using natural products such as microbesor plant extracts. For instance, using various microorganisms such as bacteria, fungi, algae, yeast and so on, it can be synthesized extracellularly or intracellularly. For this approach, in the required growth medium, microorganism which helps in the synthesis of nanoparticles is mixed with solution containing metal precursor. Then it is incubated for the synthesis of nanoparticles. Nanoparticles can also be synthesized using plant extract, by mixing metal precursor with the extract. Then incubating it for required period of time, at room or boiling temperature along with exposure of light [14].

\subsection{Nanotechnology in Cancer}

Nanoparticles are widely used for the treatment of many diseases, but cancer treatment using nanoparticles is the most prominent [5]. Cancer is one of the challenging diseases, brain cancer is most challenging among different types of cancer. It cannot be easily detected and treated because it is difficulty associated with imaging and entry of therapeutic agents through the blood brain barrier and then into the brain. But nanotechnology has helped in overcoming these difficulties by crossing the blood-brain barrier and then releasing the nanomaterials bound anti-cancer drugs such as loperamide and doxorubicin at therapeutic concentrations [13]. For the treatment of cancer, iron nanoparticles or gold shells are commonly used [1].

A popular anti-cancer drug, Paclitaxel is used to treat various types of cancer such as ovarian, skin, lung and esophageal cancer. The mode of action of this drug is by destabilizing microtubule and as a result leading to apoptosis. This is a water insoluble drug and hence it administered as solution in ethanol along with a solvent, polyoxyethylated castor oil. But this approach is associated with side effects, hence premedications such as administration of steroids and antihistamines are required. Different versions of paclitaxel (drug) loaded nanoparticles have shown different levels of efficacy. Hence to improve the performance of the drug, different nanoparticle-based drug delivery strategies can be employed $[1,9,10]$.

\subsection{Nanotechnology in Angiogenesis}

Aggressive growth of tumor occurs due to angiogenesis. Hence for cancer treatment, it is necessary to inhibit angiogenesis using antiangiogenic agents. A group of researchers (Sengupta and colleagues) have clearly described that disruption of blood vessels of tumor has great impact on drug (chemotherapeutic agent) delivery [10]. Regulation of angiogenesis is mediated by integrin $\alpha v \beta 3$ and Vascular endothelial growth factors (VEGF). Therefore these mediators can be targeted using nanoparticles, as a result inhibiting angiogenesis. In this novel anti-angiogenesis strategy, RGDSK self-assembling rosette nanotubes (RGDSK-RNT) loaded with FITC-GRGDS was used for monitoring and destroying blood vessels surrounding the tumor. Hence these RNTs can be successfully employed to control the disease progression, by targeting cell adhesion molecules and vascular endothelial growth factor [13].

\subsection{Nanotechnology in HIV/AIDS}

A potential target for the treatment of HIV/AIDS is HIV-1 Tat protein. Vaccines are developed against this candidate. But delivery of HIV-1 protease inhibitor, CGP 70726, is difficult because of its poor water solubility. Hence $\mathrm{pH}$ sensitive nanoparticles emulsified with CGP 70726 and benzyl alcohol was used for the delivery of HIV-1 protease inhibitor. It was successfully administered to dogs and analysis of blood samples showed successful drug release. SLNs (Solid Lipid Nanoparticles) loaded with DNA and Tat peptide was administered to the lungs of mice and results showed successful transfection but with some DNA degradation. Compared to successful drug delivery achieved in cancer and neurodegenerative disease treatment, drug delivery in the case of HIV/AIDS is less. But by conducting more in vitro studies in this area, this disproportionate focus can be addressed [10].

\subsection{Nanotechnology in Respiratory diseases}

Nanoparticles have not been widely used in drug delivery process for the treatment of respiratory diseases. But there are many examples for therapies in literature for the treatment of infectious respiratory diseases such as tuberculosis (TB), allergic and genetic respiratory diseases [10]. One of the promising approach for the treatment of TB is nanotechnology. This is because anti-TB drugs can be encapsulated and delivered successfully with the help of nanotechnology [1]. Apart from the drug delivery, various other strategies such as in the case of allergic asthma, liposome-based nanoparticle system or chitosan/interferon- $\gamma$ pDNA nanoparticles has proved to inhibit inflammation associated with respiratory diseases. In the case of patients suffering from asthma, there is a drop in production interferon- $\gamma$ (IFN- $\gamma$ ) which makes the patients prone to airway inflammation and hyperresponsiveness. Hence by supplying IFN- $\gamma$ with the help of nanoparticles as mentioned above, it is possible to increase the IFN- $\gamma$ which has dropped due to asthma [10].

\subsection{Nanotechnology in Neurodegenerative diseases}

Nanotechnology has wide application in the treatment of neurodegenerative disorders. Various nanocarriers used for the delivery of CNS therapeutics include dendrimers, nano gels, nano emulsions, liposomes, polymeric nano particles, solid lipid nano particles and nano suspensions have been studied extensively [1]. 


\section{International Journal of Engineering Applied Sciences and Technology, 2020 \\ Vol. 5, Issue 7, ISSN No. 2455-2143, Pages 198-209 \\ Published Online November 2020 in IJEAST (http://www.ijeast.com)}

\begin{abstract}
Alzheimer's disease: Alzheimer's disease (AD) is the most common form of dementia worldwide which affects more than 35 million people. Advances in nanotechnology are exerting a significant impact in neurology. These approaches, which are often based on the design and engineering of a plethora of nanoparticulate entities with high specificity for brain capillary endothelial cells, are currently being applied to early $\mathrm{AD}$ diagnosis and treatment. In addition, nanoparticles (NPs) with high affinity for the circulating amyloid- $\beta(A \beta)$ forms induce "sink effect" and it improves the $\mathrm{AD}$ condition. In vitro diagnostics for $\mathrm{AD}$ has advanced due to ultrasensitive NP-based bio-barcodes and immune sensors, as well as scanning tunneling microscopy procedures capable of detecting $A \beta 1-40$ and $A \beta 1-42[1,18]$.
\end{abstract}

Parkinson's disease: Parkinson's disease (PD) is the second most common neurodegenerative disease after Alzheimer's disease and affects one in every 100 persons above the age of 65 years. PD is a disease of the central nervous system; neuroinflammatory responses are involved and leads to severe difficulties with body motions. The present day therapies aim to improve the functional capacity of the patient for as long as possible but cannot modify the progression of the neurodegenerative process. Aim of applied nanotechnology is regeneration and neuroprotection of the central nervous system (CNS). Efforts are being taken to develop novel technologies that directly or indirectly helps in providing neuroprotection or a permissive environment and active signaling cues for guided axon growth. In order to minimize the peripheral side-effects of conventional forms of Parkinson's disease therapy, research is focused on the design, biometric simulation and optimization of an intracranial nano-enabled scaffold device (NESD) for the site-specific delivery of dopamine to the brain, as a strategy [1].

\subsection{Nanotechnology in Ophthalmology}

Ophthalmology is a branch of medicine which deals with the diagnosis and treatment of eye disorders. Some applications of nanotechnology to ophthalmology are treatment of oxidative stress; measurement of intraocular pressure, to prevent scars after glaucoma surgery, and for treatment of retinal degenerative disease using gene therapy, prosthetics, theragnostics, use of nano particles for treatment of choroidal new vessels, and regenerative nano medicine. A novel Nanoscale Dispersed eye ointment (NDEO) has been successfully developed for the treatment of severe evaporative dry eye. Several research shows applications of various nanoparticulate systems like microemulsions, nanosuspensions, nanoparticles, liposomes, niosomes, dendrimers and cyclodextrins in the field of ocular drug delivery [1].

\subsection{Nanotechnology and Antibiotic resistance}

Nano particles can be used to decrease antibiotic resistance in combination therapy. It was reported that Zinc Oxide nanoparticles have the ability to decrease the antibiotic resistance and enhance the antibacterial activity of Ciprofloxacin against microorganism, by interfering with various proteins that are interacting in the antibiotic resistance or pharmacologic mechanisms of drugs [1].

\subsection{Nanotechnology and immune response}

The nano device bucky balls can be used to alter the allergy/ immune response. These bucky balls prevent mast cells from releasing histamine into the blood and tissues, as they bind to free radicals better than any anti-oxidant available, such as vitamin $\mathrm{E}$ [1].

\subsection{Nanodentistry}

Nanotechnology plays an important role in dentistry. Nanodentistry helps in the maintenance of comprehensive oral health through the use of nanomaterials, biotechnology (inclusive tissue engineering) and dental nanorobotics [2]. Various nanomaterials can be used for restoration of decayed, carious, missing and fractured teeth. Advancement in nanotechnology has brought nanocomposites, nanoimpression and nanoceramic into the domain of clinical dentistry. Strength and esthetics are the two important properties possessed by composite materials. Since Polymethyl methacrylate (PMMA) possess good optical properties, biocompatibility, easy processing, and reparability, it has been used for almost all of the removable dental prostheses. But PMMA has few limitations such as poor strength, low fracture resistance, andmicrobial adhesion. In order to overcome these limitations, shortcoming nanostructuring materials such as $\mathrm{TiO}_{2}$ and $\mathrm{Fe}_{2} \mathrm{O}_{3}$ are incorporated in the polymethylmethacrylate. Ceramics have been used in prosthodontics in the manufacture of dental crowns. Nanocomposite artificial teeth has also been developed [21].

\subsection{Nanotechnology in visualisation}

Drug distribution and its metabolism can be studied by tracking movement. Cells are dyed to track their movement throughout the body and they are excited by light of a certain wavelength to glow. Luminescent tags are used to dye various numbers of cells. These luminescent tags are quantum dots attached to proteins which can penetrate cell membranes. The dots can be of various sizes. Sizes are selected in such a way that the frequency of light used makes a group of quantum dots fluoresce and make another group incandesce. Thus both groups can be lit using a single light source [1].

\subsection{Nanosurgery}

A new concept in surgical medicine-Nanosurgery. An outcome of present surgery is a small wound. A small 


\section{International Journal of Engineering Applied Sciences and Technology, 2020 \\ Vol. 5, Issue 7, ISSN No. 2455-2143, Pages 198-209 \\ Published Online November 2020 in IJEAST (http://www.ijeast.com)}

surgical wound implies less blood loss and minimisation the complication of intraoperative and postoperative. Advancement in nanomedicine has shown that nano-surgery could be performed with submicrometer precision and without visible damage to the cell. Targeted nano-therapy based on nano-pharmacology is found to be valuable in nanosurgery [1]. Surgical nanorobots can be used in nanosurgery. They can be introduced into the body the vascular system or at the ends of catheters into various vessels and other cavities in the body. These surgical nanorobot are guided or programmed by human surgeons [19]. Nanoneedles and nanotweezers are being developed which helps in making cell surgery a possibility in the near future. Suture needles incorporating nanosized stainless steel crystals are being developed which helps in making incisions at cell-level [21].

\subsection{Nanotechnology and Gene therapy}

Gene therapy is defined as administration of a nucleic acid construct into the cell which can treat or prevent the diseases. It also aims at curing diseases via changing the expression of genes that are responsible for the pathological case. Currently many chronic diseases are found to be cured by gene therapy. Two important aspect of gene therapy are: (1) Efficient and safe delivery of genes to the target cell in vitro and in vivo;(2) Effective monitoring of modified cells or modifying agents by noninvasive imaging techniques[20]. Nanobiotechnology plays a very important role in gene therapy. Biodegradable and non-biodegradable inorganic particles can be fabricated on the nano-scale so that it can bind DNA, can be internalized across the plasma membrane and finally released into the cytoplasm for a protein final expression[2].

\subsection{Nanotechnology in cardiac therapy}

Cardiac diseases are the major cause of mortality and morbidity in human beings[19]. One of the fundamental and unresolved problems in cardiovascular biology is the in vivo detection of atherosclerotic disease and the evaluation of atherosclerotic disease activity[6]. Oral and systemic administration of drugs does not provide appropriate therapeutic drug levels in the target arteries for sufficient periods of time. Even though, biomedical engineers have already succeeded in developing microscale instruments to open blocked arteries and to treat other cardiovascular diseases. But these tools are bulky and infection prone. Nanotechnology-based tools can be effectively used to treat the cardiovascular diseases[19]. Nanosystems have been applied to the area of atherosclerosis, thrombosis, and vascular biology [6].

\section{ENVIRONMENTAL AND SOCIETAL CONSIDERATIONS}

Although nanotechnology has wide range of applications in the field of medicine, the effect of nanomaterials on environment and public health is poorly understood. In recent years, to help in the further expansion of this field by reducing the risks and increasing the potential benefits, there is need for data regarding the different kinds of risk associated with the nanoparticles $[2,5,10]$. The complete nanoparticles or their degraded components are associated with various symptoms such as dermal changes, neural, cardiac and growth toxicity. Hence it is necessary to monitor and collect data regarding the long term toxic effects of nanoparticles. Biodegradable nanopolymers (PLA, PGA,PLGA based nanoparticles) used for the delivery of anti-cancer drugs, can be fully dissolved to lower the toxicity effects. It is necessary for the nanoparticles to overcome the cleansing forces such as immune system, kidney and liver in the body which consider nanoparticles as aliens in the blood stream. Nanoparticles are engulfed and degraded by the single-core phagocytic system (phagocytotic cells in liver, spleen and bone marrow) [2].

\subsection{Potential risks of Nanomedicine}

In order to assess the risk factors, first and foremost, it is necessary to understand the physicochemical properties pertaining to nanocarriers and nanomedicine. Physicochemical properties such as size, shape, hydrophobicity and hydrophilicity, degradability, catalytic ability and so on are directly related to the in vivo biological fate and behavior of the nanoparticles. The transport and distribution of non-biological complex drugs (NBCDs) depend on the blood proteins (albumin, transferring, immunoglobulin, fibrinogen and so on) that get adsorbed to the nanocarriers and nanomedicine injected into the blood. In addition to this nanomedicine are quickly uptaken by cells of the reticuloendothelial system. Therefore this result in altered biokinetics which leads to unpredictable effects such as high concentrations of nanoparticles at particular parts of the body (liver, spleen) [5,6].

\subsection{Reduction of side effects via Safe-By-Design approaches}

Secondly, it is necessary to develop nanotherapeutics rationally by considering their therapeutic efficacy and safety, from conception to implementation. Nanomaterials containing degradable components and that has been already approved safe by the FDA should be preferred for desingning. Hazardoungs nanomaterial properties such as high cationic density, high surface reactivity and so on should be considered while designing. This can be done by subjecting the different components of the nanoparticle to multiparametric high-throughput screening, which helps in reducing the in vitro toxic effects such as organellar damage, biopersistence and so on. Attempts are being made to correlate the adverse in vitro effects with specific physicochemical properties. This approach also helps in reducing the number of animal studies to a certain level, but ultimately the efficacy and safety of the design should be analyzed at in vivo conditions only [5]. 


\section{International Journal of Engineering Applied Sciences and Technology, 2020 Vol. 5, Issue 7, ISSN No. 2455-2143, Pages 198-209 \\ Published Online November 2020 in IJEAST (http://www.ijeast.com)}

\section{ETHICS}

Science offers various fascinating areas of research. One among them is science of nanomedicine (Nanotechnology) which has gained a lot of attention in the past two decades. This is because a lot of research has been conducted in this field which led to successful completion of various clinical trials and filling of nearly 1500 patents [14]. Thus it is clear that in the coming decade nanotechnology applications will widen further and gain a priority in medicine and medical technology [3]. At the same time it is necessary to carefully analyze the ethical, legal and social aspects of this emerging technology. In this review, for instance let us consider the standards and regulations set by the ethics committees at the European scale. Hence it is clear that in depth-analysis is required based on the following principles [4]:

$\checkmark$ Non-instrumentalisation: Individuals should not be treated as means instead as an end of their own (solely depend on their inherent value in such a way that it does not depend on anything else)

$\checkmark$ Privacy: Confidentiality should be maintained, that a person's right to privacy should be not be invaded

$\checkmark$ Non-discrimination: Regardless to their sex, race, color, language and several other discriminations, every person deserves equal treatment. Unless if there are reasons to justify the difference in treatment.

$\checkmark$ Informed consent: It is a must to get prior permission from the person before any medical or research intervention

$\checkmark$ Equity: Every person should have a fair access to the benefits under consideration

$\checkmark$ The Precautionary Principle: This principle demands the moral duty of continuous risk assessment of the new technologies.

$\checkmark$ The last principle is much of importance with reference to this context [4].

\section{CONCLUSION}

From this review it is evident that nanoparticles have proven to be a promising tool in drug and gene delivery, biomedical imaging, diagnostics biosensors, cancer therapies, tissue engineering and so on. Nanotechnology has a very bright future because of its ability to intertwine with biology-based technologies, leading to emergence of potential, innovative hybrid technologies for the welfare of human life in many aspects. In recent years, nanomedicine has gained much interest because of it potential to deliver drugs specifically to the target cells with the help of nanobots and thereby reduces damage to the healthy cells. For the delivery of drug and gene, different nanomaterials are being analysed for their physicochemical and biological properties, so that nanocarries can get smarter. Owing to their unique properties, nanomaterials (nanosensors) have the ability to produce exceptional images of tumors. Although nanomedicine has advanced a lot, many of these advances are for the diagnosis and treatment of cancer. This disproportionate focus is foresighted to be resolved with the help of research focusing on all fields of medicine in the upcoming years.

The downside of nanomedicine is the poor understanding of toxic effects of nanoparticles biocompatibility, targeting efficacy and long-term stability of nanoparticles. Hence to overcome these challenges, nanomaterials are being synthesized with the help of biological components using safe by design approach and specifically targeted to the site of action. This helps in the reduction of side effect and increases the bioavailability and efficacy of the drug. It can be concluded that nanomedicine can drastically improve the quality of life of patients besides the issues such as risks, sociological and ethical questions pertaining to it. By addressing the issues through analysis, it can be foresighted that nanomedicine can lead to revolution in the world of medicine.

\section{REFERENCES}

[1] Nikalje, A. P..(2015) "Nanotechnology and its Applications in Medicine." Medicinal Chemistry 5; 1-9.

[2] Abdussalam-Mohammed, W. (2019). Review of Therapeutic Applications of Nanotechnology in Medicine Field and its Side Effects. Journal of Chemical Reviews, 1(3), 243-251. doi: 10.33945/SAMI/JCR.2019.3.5

[3] Krukemeyer, M.G., Krenn, Hübner, F., Wagner, W., \& Resch, R. (2015). History and Possible Uses of Nanomedicine Based on Nanoparticles and Nanotechnological Progress. Journal of Nanomedicine \& Nanotechnology, 6, 1-7. https://doi.org/10.4172/21577439.1000336

[4] Patrick Boisseau a,.,Bertrand Loubaton.(2011). Nanomedicine, nanotechnology in medicine, C. R. Physique 12, 620-636. https://doi.org/10.1016/j.crhy.2011.06.001

[5] Pelaz, B., Alexiou, C., Alvarez-Puebla, R. A., Alves, F., Andrews, A. M., Ashraf, S., Balogh, L. P., Ballerini, L., Bestetti, A., Brendel, C., Bosi, S., Carril, M., Chan, W. C., Chen, C., Chen, X., Chen, X., Cheng, Z., Cui, D., Du, J., Dullin, C., Parak, W. J. (2017). Diverse Applications of Nanomedicine. ACS nano, 11(3), 2313-2381. https://doi.org/10.1021/acsnano.6b06040

[6] Zuo, L., Wei, W., Morris, M., Wei, J., Gorbounov, M., \& Wei, C. (2007). New technology and clinical applications of nanomedicine. The Medical clinics of North America, 91(5), 845-862. https://doi.org/10.1016/j.mcna.2007.05.004

[7] Gulati, Neha\& Gupta, Himanshu. (2012). Nanomedicine: Potential Devices for Diagnostics. Recent Patents on 
International Journal of Engineering Applied Sciences and Technology, 2020

Vol. 5, Issue 7, ISSN No. 2455-2143, Pages 198-209

Published Online November 2020 in IJEAST (http://www.ijeast.com)

Nanomedicine.https://doi.org/10.2174/18779123112020201 46

[8] Shrivastava, S.S., \& Dash, D. (2009). Applying Nanotechnology to Human Health: Revolution in Biomedical Sciences. Journal of Nanotechnology, 2009, 114.vhttps://doi.org/ 10.1155/2009/184702

[9] Srilatha B (2016). A review on Nanotechnology and nanomedicine. journal of pharmaceutics and Nanotechnology, 7.

[10] Murthy S. K. (2007). Nanoparticles in modern medicine: state of the art and future challenges. International journal of nanomedicine, 2(2), 129-141.

[11] Andreou, C., Pal, S., Rotter, L., Yang, J., \&Kircher, M. F. (2017). Molecular Imaging in Nanotechnology and Theranostics. Molecular imaging and biology, 19(3), 363372. https://doi.org/10.1007/s11307-017-1056z

[12] Kim, J., Lee, N., \&Hyeon, T. (2017). Recent development of nanoparticles for molecular imaging. Philosophical transactions. Series A, Mathematical, physical, and engineering sciences, 375(2107), 20170022. https://doi.org/10.1098/rsta.2017.0022

[13] Suri, Sarabjeet\&Fenniri, Hicham\& Singh, Baljit. (2007). Nanotechnology-based Drug Delivery Systems. Journal of occupational medicine and toxicology (London, England). 2. 16. https://doi.org/10.1186/1745-6673-2-16

[14] Patra, J. K., Das, G., Fraceto, L. F., Campos, E., Rodriguez-Torres, M., Acosta-Torres, L. S., Diaz-Torres, L. A., Grillo, R., Swamy, M. K., Sharma, S., Habtemariam, S., \& Shin, H. S. (2018). Nano based drug delivery systems: recent developments and future prospects. Journal of nanobiotechnology, 16(1),

https://doi.org/10.1186/s12951-018-0392-8

[15] Salata O. (2004). Applications of nanoparticles in biology and medicine. Journal of nanobiotechnology, 2(1), 3. https://doi.org/10.1186/1477-3155-2-3

[16] Saeed, Khalid \& Khan, Idrees. (2017). Nanoparticles: Properties, Applications and Toxicities. Arabian Journal of
Chemistry. 12(7), https://doi.org/10.1016/j.arabjc.2017.05.011

908-931.

[17] Bayda, S., Adeel, M., Tuccinardi, T., Cordani, M., \&Rizzolio, F. (2019). The History of Nanoscience and Nanotechnology: From Chemical-Physical Applications to Nanomedicine.Molecules (Basel, Switzerland), 25(1), 112. https://doi.org/10.3390/molecules25010112

[18] Brambilla, D., Le Droumaguet, B., Nicolas, J., Hashemi, S. H., Wu, L. P., Moghimi, S. M., Couvreur, P., \& Andrieux, K. (2011). Nanotechnologies for Alzheimer's disease: diagnosis, therapy, and safety issues. Nanomedicine : nanotechnology, biology, and medicine, 7(5), 521-540. https://doi.org/10.1016/j.nano.2011.03.008

[19] Singh, S., \& Singh, A. (2013). Current status of nanomedicine and nanosurgery. Anesthesia, essays and researches, 7(2), 237-242. https://doi.org/10.4103/02591162.118976

[20] Herranz, F., Almarza, E., Rodríguez, I., Salinas, B., Rosell, Y., Desco, M., Bulte, J. W., \& Ruiz-Cabello, J. (2011). The application of nanoparticles in gene therapy and magnetic resonance imaging. Microscopy research and technique, 74(7), 577-591. https://doi.org/10.1002/jemt.20992

[21] Aeran, H., Kumar, V., Uniyal, S., \&Tanwer, P. (2015). Nanodentistry: Is just a fiction or future. Journal of oral biology and craniofacial research, 5(3), 207-211. https://doi.org/10.1016/j.jobcr.2015.06.012

[22] Sundar, S., Kundu, J., \&Kundu, S. C. (2010). Biopolymeric nanoparticles. Science and technology of advanced materials, $11(1), \quad 014104$. https://doi.org/10.1088/1468-6996/11/1/014104

[23] Watkins, R., Wu, L., Zhang, C., Davis, R. M., \&Xu, B. (2015). Natural product-based nanomedicine: recent advances and issues. International journal of nanomedicine, 10, 6055-6074. https://doi.org/10.2147/IJN.S92162 